Discrete Comput Geom 28:255-273 (2002)

DOI: $10.1007 / \mathrm{s} 00454-002-2779-3$

Geometry

\title{
Random Spaces Generated by Vertices of the Cube
}

\author{
Apostolos Giannopoulos and Marianna Hartzoulaki \\ Department of Mathematics, University of Crete, \\ Iraklion, Crete, Greece \\ \{apostolo,hmarian\}@math.uch.gr
}

\begin{abstract}
Let $E_{2}^{n}=\{-1,1\}^{n}$ be the discrete cube in $\mathbb{R}^{n}$. For every $N \geq n$ we consider the class of convex bodies $K_{N}=\operatorname{co}\left\{ \pm x_{1}, \ldots, \pm x_{N}\right\}$ which are generated by $N$ random points $x_{1}, \ldots, x_{N}$ chosen independently and uniformly from $E_{2}^{n}$. We show that if $n \geq n_{0}$ and $N \geq n(\log n)^{2}$, then, for a random $K_{N}$, the inradius, the volume radius, the mean width and the size of the maximal inscribed cube can be determined up to an absolute constant as functions of $n$ and $N$. This geometric description of $K_{N}$ leads to sharp estimates for several asymptotic parameters of the corresponding $n$-dimensional normed space $X_{N}$.
\end{abstract}

\section{Introduction}

The use of random spaces in the study of finite-dimensional normed spaces provided a way of proving the existence of spaces or operators with extremal properties. Several important problems of the theory were solved by introducing a suitable probability space consisting of $n$-dimensional spaces and showing that random selection of its elements gives objects with the desired properties. Among many existence results proved in this way, we mention the existence of a pair of $n$-dimensional spaces with Banach-Mazur distance of order as large as $n$ [18], the existence of a space whose unconditional basis constant [15] or basis constant [33], [19] has order as large as $\sqrt{n}$, the existence of a space whose Banach-Mazur distance to $\ell_{\infty}^{n}$ is greater than $c \sqrt{n} \log n$ [34]. In particular, random $n$-dimensional subspaces of $\ell_{\infty}^{N}$ with $N=\lambda n, \lambda>1$ (i.e. spaces whose unit balls are random sections of the cube $Q_{N}$ of dimension proportional to $N$ ), provided examples of spaces which exhibited pathology with respect to various asymptotic parameters of the theory: this line of thought has its origin in [23] and [16] (see also [32], [35], [13] and the articles mentioned above).

In this article we consider convex hulls of random subsets of the set of vertices of the cube and the class of random spaces they generate. In order to define our probability space precisely, we consider the discrete cube $E_{2}^{n}=\{-1,1\}^{n}$ in $\mathbb{R}^{n}$ equipped with the 
uniform probability measure, and fix $N \geq n$. Next, we consider $N$ independent random points $x_{1}, \ldots, x_{N}$ uniformly distributed over $E_{2}^{n}$, and for every choice $x_{1}, \ldots, x_{N}$, we write $M_{N}$ for the convex hull

$$
M_{N}:=M\left(x_{1}, \ldots, x_{N}\right)=\operatorname{co}\left\{x_{1}, \ldots, x_{N}\right\}
$$

and $K_{N}$ for the absolute convex hull

$$
K_{N}:=K\left(x_{1}, \ldots, x_{N}\right)=\operatorname{co}\left\{ \pm x_{1}, \ldots, \pm x_{N}\right\} .
$$

The symmetric convex body $K_{N}$ (if non-degenerate) induces a norm on $\mathbb{R}^{n}$. We write $X_{N}$ for the normed space whose unit ball is $K_{N}$. In this way, for every $N \geq n$ we obtain a class of random $n$-dimensional spaces, which we denote by $\mathcal{B}_{N}$. The dual space of $X_{N}$ is denoted by $X_{N}^{*}$ and the class of dual spaces by $\mathcal{B}_{N}^{*}$.

Section 2 is devoted to the study of the geometry of a random $K_{N}$. We say that a property $(P)$ holds for a random $K_{N}$ if the probability of the $N$-tuples $\left(x_{1}, \ldots, x_{N}\right)$ for which $K_{N}$ has $(P)$ is greater than $1-\exp (-n)$ (it tends to 1 "exponentially" as the dimension $n$ grows to infinity). There are three basic sources of information on a random $K_{N}$, depending on the number $N$ of vertices. First, we prove that if $N \geq \lambda_{0} n$ where $\lambda_{0}>1$ is a fixed constant, then (with high probability) $K_{N}$ contains a centered ball of radius independent of $n$ and $N$.

Fact 1. If $N \geq \lambda_{0} n$, then $K_{N} \supseteq c_{1} B_{2}^{n}$ with high probability, where $B_{2}^{n}$ is the Euclidean unit ball and $\lambda_{0}>1, c_{1}>0$ are absolute constants.

Since $K_{N} \subseteq Q_{n}:=[-1,1]^{n}$, this estimate is clearly optimal, the interesting point being that it starts being true for a random $K_{N}$ when $N$ is as low as of the order of $n$. The proof of this fact is a consequence of the observation that a random "small" set of vertices of the cube is already enough to substitute $E_{2}^{n}$ in the classical Khintchine inequality: for all $a_{1}, \ldots, a_{n} \in \mathbb{R}$,

$$
\frac{1}{|A|} \sum_{\varepsilon \in A}\left|\varepsilon_{1} a_{1}+\cdots+\varepsilon_{n} a_{n}\right| \simeq\left(a_{1}^{2}+\cdots+a_{n}^{2}\right)^{1 / 2},
$$

if $m \geq \lambda_{0} n$ and $A$ is a random subset of $E_{2}^{n}$ of cardinality $|A|=m$.

Our second main result is that if $N \geq n(\log n)^{2}$, then $K_{N}$ contains (with high probability) a centered cube whose edges have length $\sqrt{\log (N / n)} / \sqrt{n}$.

Fact 2. There exists $n_{0} \in \mathbb{N}$ with the following property: if $n \geq n_{0}$ and $N \geq n(\log n)^{2}$, then

$$
K_{N} \supseteq c\left(\sqrt{\log (N / n)} B_{2}^{n} \cap Q_{n}\right) \supseteq\left(c_{2} \sqrt{\log (N / n)} / \sqrt{n}\right) Q_{n}
$$

with probability greater than $1-e^{-n}$, where $c_{2}>0$ is an absolute constant.

In the range $N \geq \exp \left((\log n)^{2}\right)$, the first inclusion was recently proved by Bárány and Pór in [4] (see the remarks after Theorem 2.2). Fact 2 should be compared with the following result which was proved in [17]: There exists a constant $\kappa>0$ with the 
following property: for every $\delta \in(0,1)$ and every convex body $K$ with centroid at the origin in $\mathbb{R}^{n}, N \geq c(\delta) n^{\kappa}$ points $x_{1}, \ldots, x_{N}$ chosen uniformly and independently from $K$ satisfy with probability greater than $1-\delta$,

$$
K \supseteq M_{N}=\operatorname{co}\left\{x_{1}, \ldots, x_{N}\right\} \supseteq \frac{c \log N}{n} K
$$

where $c>0$ is an absolute constant. Fact 2 may be viewed as a discrete version of the case $K=Q_{n}$ in the above result. The argument in [17] makes essential use of the Brunn-Minkowski inequality: the main point there is that the $L_{\psi_{1}}$-norm of linear functionals on convex bodies is bounded by their $L_{1}$-norm. Note that the dependence on $N$ in Fact 2 is better (this reflects the fact that linear functionals $f$ on the cube satisfy the stronger inequality $\|f\|_{L_{\psi_{2}}} \leq c\|f\|_{L_{1}}$ ).

Finally, we observe that combining Fact 2 with well-known volume estimates from [20] and [9] one can determine the volume radius of $K_{N}$ and $K_{N}^{\circ}$ up to an absolute constant.

Fact 3. If $N \geq n(\log n)^{2}$, then for a random $K_{N}$ we have

(a) $\left|K_{N}\right|^{1 / n} \simeq \sqrt{\log (N / n)} / \sqrt{n}$ and $\left|K_{N}^{\circ}\right|^{1 / n} \simeq 1 / \sqrt{n \log (N / n)}$,

(b) $w\left(K_{N}\right) w\left(K_{N}^{\circ}\right) \leq c_{3} \sqrt{\log n} \sqrt{\log N} / \sqrt{\log (N / n)}$,

where $w(\cdot)$ denotes mean width and $c_{3}>0$ is an absolute constant.

Combining all three facts we have a precise description of the unit ball of $X_{N}$. A random $K_{N}$ belongs to a rather restricted class of convex bodies for which many asymptotic parameters can be estimated through known methods. This is done in Section 3, where we start by studying unconditionality properties of $X_{N}$ as a function of $N$. Our first result concerns the Banach-Mazur distance from a random $X_{N}$ to the class $\mathcal{U}$ of spaces with 1-unconditional basis.

Fact 4. For every $\delta \in(0,1)$ we can find $c(\delta)=O\left(\log \left(\delta^{-1}\right)\right)$ such that: if $N \geq c(\delta) n$, then $X_{N} \in \mathcal{B}_{N}$ satisfies

$$
d\left(X_{N}, \mathcal{U}\right) \geq \frac{c_{4} \sqrt{n}}{\sqrt{\log (2 N / n)}}
$$

with probability greater than $1-\delta$, where $c_{4}>0$ is an absolute constant and denotes Banach-Mazur distance.

Fact 2 shows that when $N \geq n(\log n)^{2}$, then $d\left(X_{N}, \mathcal{U}\right)$ is "attained" for $\ell_{\infty}^{n}$ and has exactly the order given by Fact 4 . Also, for suitable $N \simeq n$, Fact 4 shows the existence of a space whose distance from $\mathcal{U}$ is of the maximal possible order $\sqrt{n}$ (this is a well-known fact; see [15]).

Facts 1 and 3(b) show that the Euclidean ball is "equivalent" to the distance and $\ell$ ellipsoid of $K_{N}$. Thus, although the unconditional basis constant of $X_{N}$ is large, we may apply the method of random orthogonal factorizations to obtain upper estimates for the Banach-Mazur distance from $X_{N}$ to special classes of spaces. In particular, we prove 
the following:

Fact 5. For every $N \geq n$ and for a random $X_{N}, d\left(X_{N}, X_{N}^{*}\right) \leq C \sqrt{n \log n}$ where $C>0$ is an absolute constant.

Finally, we obtain estimates for the isotropic constant of the unit balls of spaces in the classes $\mathcal{B}_{N}$ and $\mathcal{B}_{N}^{*}$. For a random $K_{N}^{\circ}, N \geq n(\log n)^{2}$, the isotropic constant is bounded by an absolute constant.

Fact 6. There exist absolute constants $c, C>0$ with the following property:

(a) If $n \leq N \leq n(\log n)^{2}$, then $L_{K_{N}^{\circ}} \leq c \sqrt{\log (2 N / n)} \leq C \sqrt{\log \log n}$.

(b) If $N \geq n(\log n)^{2}$, then $L_{K_{N}^{\circ}} \leq C$ for a random $K_{N}^{\circ}$.

Some estimates for the isotropic constant of a random $K_{N}$ may be given as well.

Fact 7. Let $N \geq n(\log n)^{2}$. For a random $K_{N}$ we have

$$
L_{K_{N}} \leq C \frac{\min \{\log N, \sqrt{n}\}}{\sqrt{\log (N / n)}}
$$

where $C>0$ is an absolute constant.

Notation. We will be working in $\mathbb{R}^{n}$, which is equipped with the Euclidean structure $\langle\cdot, \cdot\rangle$. All $n$-dimensional normed spaces in this paper are of the form $X=\left(\mathbb{R}^{n},\|\cdot\|\right)$. The unit ball of $X$ is a centrally symmetric convex body in $\mathbb{R}^{n}$ which is denoted by $B_{X}$. Conversely, every centrally symmetric convex body $K$ induces the norm $\|x\|_{K}=$ $\min \{\lambda \geq 0: x \in \lambda K\}$ to $\mathbb{R}^{n}$, and $K$ is the unit ball of $X_{K}=\left(\mathbb{R}^{n},\|\cdot\|_{K}\right)$. The dual norm is defined by $\|y\|_{*}=\max \left\{|\langle x, y\rangle|: x \in B_{X}\right\}$, and the unit ball of $X^{*}=\left(\mathbb{R}^{n},\|\cdot\|_{*}\right)$ is the polar body $B_{X^{*}}=B_{X}^{\circ}$ of $B_{X}$.

We write $B_{2}^{n}$ and $S^{n-1}$ for the Euclidean unit ball and sphere, respectively, and

$$
\|x\|_{p}=\left(\sum_{i=1}^{n}\left|x_{i}\right|^{p}\right)^{1 / p}
$$

for the $\ell_{p}^{n}$-norm of $x=\left(x_{1}, \ldots, x_{n}\right) \in \mathbb{R}^{n}, 1 \leq p<\infty$ (in the case $p=\infty,\|x\|_{\infty}=$ $\left.\max _{i \leq n}\left|x_{i}\right|\right)$. The rotationally invariant probability measure on $S^{n-1}$ is denoted by $\sigma$. We use the notation $|A|$ for the volume of a convex body and for the cardinality of a finite set.

The support function of a convex body $K$ is defined by $h_{K}(y)=\max _{x \in K}\langle x, y\rangle$. The mean width of $K$ is the quantity

$$
w(K)=\int_{S^{n-1}}\left[h_{K}(\theta)+h_{K}(-\theta)\right] \sigma(d \theta)=2 \int_{S^{n-1}} h_{K}(\theta) \sigma(d \theta) .
$$

Let $X$ and $Y$ be two $n$-dimensional normed spaces. Their Banach-Mazur distance $d(X, Y)$ is defined by

$$
d(X, Y)=\min \left\{\|T\| \cdot\left\|T^{-1}\right\| \mid T: X \rightarrow Y \text { an isomorphism }\right\} .
$$


John's theorem [21] shows that $d\left(X, \ell_{2}^{n}\right) \leq \sqrt{n}$ for every $X$. It follows that $d(X, Y)$ is always bounded by $n$. On the other hand, as we already mentioned, Gluskin [18] proved that there exists an absolute constant $c>0$ such that for every $n$ one can find $n$-dimensional spaces $X_{n}, Y_{n}$ with $d\left(X_{n}, Y_{n}\right) \geq c n$.

The letters $c, c_{1}, c_{2}, c^{\prime}$, etc., are reserved for absolute positive constants, which may change from line to line. Wherever we write $a \simeq b$, this means that there exist absolute constants $c_{1}, c_{2}>0$ such that $c_{1} a \leq b \leq c_{2} a$. We refer the reader to the books [26], [30] and [37] for basic facts that we use throughout the text.

\section{Geometry of the Unit Ball}

As was mentioned in the Introduction, we say that a random $K_{N}$ has a certain property $(P)$ if

$$
\operatorname{Prob}\left(\left(x_{1}, \ldots, x_{N}\right) \in E_{2}^{n} \times \cdots \times E_{2}^{n}:(P) \text { holds for } K_{N}\right) \geq 1-e^{-n},
$$

where $K_{N}=\operatorname{co}\left( \pm x_{1}, \ldots, \pm x_{N}\right)$. In this section we give a description of the unit ball $K_{N}$ of a random element of $\mathcal{B}_{N}$ :

Theorem A. There exists $n_{0} \in \mathbb{N}$ such that if $n \geq n_{0}$ and $N \geq n(\log n)^{2}$, then a random $K_{N}$ has the following properties:

(a) $K_{N} \supseteq c_{1} B_{2}^{n}$,

(b) $K_{N} \supseteq\left(c_{2} \sqrt{\log (N / n)} / \sqrt{n}\right) Q_{n}$,

(c) $\left|K_{N}\right|^{1 / n} \simeq \sqrt{\log (N / n)} / \sqrt{n}$ and $\left|K_{N}^{\circ}\right|^{1 / n} \simeq 1 / \sqrt{n \log (N / n)}$,

(d) $w\left(K_{N}\right) w\left(K_{N}^{\circ}\right) \leq c_{3} \sqrt{\log n} \sqrt{\log N} / \sqrt{\log (N / n)}$,

where $c_{1}, c_{2}$ and $c_{3}$ are absolute positive constants.

The proofs of these facts are presented in the next four subsections.

\subsection{Inradius of $K_{N}$}

We first show that if $N \geq c \log \left(\delta^{-1}\right) n$, then, with probability greater than $1-\delta, K_{N}$ contains a Euclidean ball of radius independent from $n$ and $N$. Our main tool is the fact that, with high probability, few vertices of the cube represent $E_{2}^{n}$ in the classical Khintchine inequality. This statement was first proved in [31] (see also [17] for the formulation we use in this paper).

Lemma 2.1. Let $\delta \in(0,1)$. If $N \geq c \log \left(\delta^{-1}\right) n$, then $N$ points $x_{1}, \ldots, x_{N}$ chosen uniformly and independently from $E_{2}^{n}$ satisfy with probability greater than $1-\delta$ the inequality

$$
c_{1}\|y\|_{2} \leq \frac{1}{N} \sum_{i=1}^{N}\left|\left\langle y, x_{i}\right\rangle\right| \leq c_{2}\|y\|_{2}
$$

for all $y \in \mathbb{R}^{n}$, where $c, c_{1}, c_{2}>0$ are absolute constants. 
In particular, Lemma 2.1 holds true with $\delta=e^{-n}$ provided that $N \geq c n^{2}$. We assume that Lemma 2.1 applies for the vertices $\pm x_{1}, \ldots, \pm x_{N}$ of $K_{N}$. Note that if $W_{1}, W_{2}$ are convex bodies, then $W_{1} \subseteq W_{2}$ if and only if $h_{W_{1}} \leq h_{W_{2}}$. By Lemma 2.1 we have

$$
\begin{aligned}
h_{K_{N}}(y) & =\max _{j \leq N}\left|\left\langle x_{j}, y\right\rangle\right| \geq \frac{1}{N} \sum_{j=1}^{N}\left|\left\langle x_{j}, y\right\rangle\right| \\
& \geq c_{1}\|y\|_{2}=c_{1} h_{B_{2}^{n}}(y)
\end{aligned}
$$

for every $y \in \mathbb{R}^{n}$, which shows that $K_{N} \supseteq c_{1} B_{2}^{n}$. Thus, we have proved the following.

Proposition 2.1. Let $\delta \in(0,1)$. If $N \geq c \log \left(\delta^{-1}\right) n$, then $K_{N} \supseteq c_{1} B_{2}^{n}$ with probability greater than $1-\delta$.

\subsection{Affine Cubes Inside $K_{N}$}

Our next aim is to show that if $n$ is big enough and $N \geq n(\log n)^{2}$, then $K_{N}$ contains (with high probability) a centered cube $P$ such that $\left|K_{N}\right|^{1 / n} \simeq|P|^{1 / n}$. This is a consequence of the following theorem.

Theorem 2.1. There exist $n_{0} \in \mathbb{N}$ and an absolute constant $c>0$ with the following property: if $n \geq n_{0}$ and $N>n(\log n)^{2}$, then $N$ random points $x_{1}, \ldots, x_{N}$ chosen independently and uniformly from $E_{2}^{n}$ satisfy, with probability greater than $1-e^{-n}$,

$$
M_{N}:=\operatorname{co}\left\{x_{1}, \ldots, x_{N}\right\} \supseteq c\left(\sqrt{\log (N / n)} B_{2}^{n} \cap Q_{n}\right),
$$

where $Q_{n}=[-1,1]^{n}$ is the unit cube in $\mathbb{R}^{n}$.

The proof makes heavy use of a theorem of Montgomery-Smith. Consider the interpolation norm

$$
K_{1,2}(x, t)=\inf \left\{\|y\|_{1}+t\|x-y\|_{2}: y \in \mathbb{R}^{n}\right\},
$$

where $x \in \mathbb{R}^{n}$ and $t>0$. We need the main result from [27].

Fact. There exists an absolute constant $r \geq 1$ such that for every $y \in \mathbb{R}^{n}$ and every $t>0$,

$$
P\left(\left\{x \in E_{2}^{n}:\langle x, y\rangle>r^{-1} K_{1,2}(y, t)\right\}\right) \geq r^{-1} \exp \left(-r t^{2}\right) .
$$

The geometric interpretation of $K_{1,2}$ is the following: Fix $\alpha>0$ and consider the symmetric convex body

$$
C(\alpha)=r^{-1}\left(\alpha B_{2}^{n} \cap Q_{n}\right) .
$$

Then the support function of $C(\alpha)$ is given by

$$
h_{C(\alpha)}(x)=r^{-1} \inf \left\{\|y\|_{1}+\alpha\|x-y\|_{2}: y \in \mathbb{R}^{n}\right\}=r^{-1} K_{1,2}(x, \alpha) .
$$


With this notation, we have:

Lemma 2.2. Let $\alpha>0$. For every $\theta \in S^{n-1}$,

$$
P\left(\left\{x \in E_{2}^{n}:\langle x, \theta\rangle \geq h_{C(\alpha)}(\theta)\right\}\right) \geq r^{-1} \exp \left(-r \alpha^{2}\right) .
$$

Let $x_{1}, \ldots, x_{N}$ be chosen independently and uniformly from $E_{2}^{n}$, and consider their convex hull $M_{N}:=M\left(x_{1}, \ldots, x_{N}\right)$. Since

$$
h_{M_{N}}(\theta)=\max _{j \leq N}\left\langle x_{j}, \theta\right\rangle,
$$

we have

$$
\begin{aligned}
P\left(h_{M_{N}}(\theta) \leq h_{C(\alpha)}(\theta)\right) & =\left(P\left(\left\{x \in E_{2}^{n}:\langle x, \theta\rangle<h_{C(\alpha)}(\theta)\right\}\right)\right)^{N} \\
& \leq\left(1-r^{-1} \exp \left(-r \alpha^{2}\right)\right)^{N} \\
& \leq \exp \left(-\frac{N}{r} \exp \left(-r \alpha^{2}\right)\right)
\end{aligned}
$$

for every $\theta \in S^{n-1}$.

Let $\delta \in(0,1)$. We choose a $\rho$-net $\mathcal{N}$ of $S^{n-1}$, with cardinality $|\mathcal{N}| \leq(1+(2 / \rho))^{n}$ (see p. 7 of [26]). Then the estimate above proves the following fact:

Lemma 2.3. Let $N \geq n$ and $\rho, \delta \in(0,1), \alpha>0$. If

$$
\left(1+\frac{2}{\rho}\right)^{n} \leq \delta \exp \left(\frac{N}{r} \exp \left(-r \alpha^{2}\right)\right)
$$

then, with probability greater than $1-\delta, N$ random points $x_{1}, \ldots, x_{N}$ chosen independently and uniformly from $E_{2}^{n}$ satisfy

$$
h_{M_{N}}(\theta) \geq h_{C(\alpha)}(\theta),
$$

for every $\theta \in \mathcal{N}$.

Proof of Theorem 2.1. Let $N \geq n(\log n)^{2}$. We choose $\alpha=(1 / 2 \sqrt{r}) \sqrt{\log (N / n)}$ and apply Lemma 2.3 with $\delta=e^{-n}:$ If

$$
1+\log \left(1+\frac{2}{\rho}\right) \leq \frac{N}{r n} \exp \left(-\frac{1}{4} \log \left(\frac{N}{n}\right)\right)=\frac{1}{r}\left(\frac{N}{n}\right)^{3 / 4},
$$

then with probability greater than $1-e^{-n}$ the convex hull $M_{N}$ of $x_{1}, \ldots, x_{N}$ satisfies

$$
h_{M_{N}}(\theta) \geq h_{C(\alpha)}(\theta)
$$

for every $\theta$ in a $\rho$-net of $S^{n-1}$.

Let $u \in S^{n-1}$. There exists $\theta \in \mathcal{N}$ such that $\|u-\theta\|_{2}<\rho$. Then

$$
\begin{aligned}
h_{M_{N}}(u) & \geq h_{M_{N}}(\theta)-h_{M_{N}}(\theta-u) \geq h_{C(\alpha)}(\theta)-h_{M_{N}}(\theta-u) \\
& \geq h_{C(\alpha)}(u)-\left[h_{C(\alpha)}(\theta-u)+\|\theta-u\|_{1}\right] .
\end{aligned}
$$


For $n$ large enough (depending on $r$ ) we have $\alpha \geq 1$. It follows that

$$
\frac{1}{r} B_{2}^{n} \subseteq C(\alpha) \subseteq \frac{\alpha}{r} B_{2}^{n}
$$

and hence

$$
h_{C(\alpha)}(\theta-u)+\|\theta-u\|_{1} \leq\left(\frac{\alpha}{r}+\sqrt{n}\right) \rho \leq 2 r \sqrt{n} \rho h_{C(\alpha)}(u) .
$$

It follows that

$$
h_{M_{N}}(u) \geq \frac{h_{C(\alpha)}(u)}{2}
$$

if we choose $\rho=1 /(4 r \sqrt{n})$. With this choice of $\alpha$ and $\rho$, it remains to check that (2.8) is satisfied for large enough $n$. The condition is equivalent to

$$
c(r) \log n \leq\left(\frac{N}{n}\right)^{3 / 4}
$$

and, since $N>n(\log n)^{2}$, this is satisfied if $(\log n)^{1 / 2}>c(r)$ which holds true for $n \geq n_{0}=\exp \left(c^{2}(r)\right)$. The theorem follows with $c=1 /\left(4 r^{3 / 2}\right)$.

Since $Q_{n} \supseteq B_{2}^{n}$, Theorem 2.1 implies Proposition 2.1 in the case $N \geq n(\log n)^{2}$. Also, since $\sqrt{n} B_{2}^{n} \supseteq Q_{n}$ we get the second part of Theorem A:

Theorem 2.2. There exist $n_{0} \in \mathbb{N}$ and an absolute constant $c>0$ with the following property: if $n \geq n_{0}$ and $N>n(\log n)^{2}$, then $N$ random points $x_{1}, \ldots, x_{N}$ chosen independently and uniformly from $E_{2}^{n}$ satisfy, with probability greater than $1-e^{-n}$,

$$
K_{N} \supseteq c \frac{\sqrt{\log (N / n)}}{\sqrt{n}} Q_{n} .
$$

Remarks. An earlier version of this paper included a self-contained and elementary proof of Theorem 2.2 for $N$ polynomial in $n$ ( $N \geq n^{\kappa}$, where $\kappa>0$ is a fixed constant). It turned out that analogous statements had already appeared in the literature.

One of the referees informed us that this result can be derived (if one goes inside the proofs) from the methods of Dyer et al. in [12]. In a very recent paper, Bárány and Pór [4] showed the existence of 0-1 polytopes with superexponential number of facets. One main step in their argument is a statement equivalent to Theorem 2.1 (Lemma 4.3 in [4]) which is proved by a refinement of the method of [12] for the range $N \geq \exp \left((\log n)^{2}\right)$.

We are grateful to a second referee for pointing out that Montgomery-Smith's theorem is also sufficient for proving Theorem 2.2. In fact, making full use of [27] one gets a proof of Theorem 2.1 for $N \geq n(\log n)^{2}$.

\subsection{Volume Estimates}

We now pass to volume estimates. Consider the polar body

$$
K_{N}^{\circ}=\left\{y \in \mathbb{R}^{n}:\left|\left\langle y, x_{i}\right\rangle\right| \leq 1, \quad i=1, \ldots, N\right\} .
$$


This is an intersection of symmetric strips, and very precise lower bounds for its volume are available (see [20], [9], [3], [2] and [8] for related results):

Lemma 2.4. There exists an absolute constant $c>0$ such that, for every $N \geq n$,

$$
\left|K_{N}^{\circ}\right|^{1 / n} \geq \frac{c}{\sqrt{n \log (2 N / n)}} .
$$

Combining this estimate with the Blaschke-Santaló inequality $\left|K_{N}\right| \cdot\left|K_{N}^{\circ}\right| \leq\left|B_{2}^{n}\right|^{2}$, we get

$$
\left|K_{N}\right|^{1 / n} \leq c^{\prime} \frac{\sqrt{\log (2 N / n)}}{\sqrt{n}} .
$$

On the other hand, Theorem 2.2 shows that if $N \geq n(\log n)^{2}$, then $K_{N}$ contains a cube of about the same volume.

Proposition 2.2. If $N \geq n(\log n)^{2}$, then a random $K_{N}$ contains a centered cube $P$ such that

$$
\left(\frac{\left|K_{N}\right|}{|P|}\right)^{1 / n} \leq C
$$

where $C>0$ is an absolute constant.

This fact shows that Theorem 2.2 is optimal in a very strong sense: a random $K_{N}$ has the maximal possible volume. It also determines the volume radius of $K_{N}$ and $K_{N}^{\circ}$ :

Proposition 2.3. If $N \geq n(\log n)^{2}$, then for a random $K_{N}$ we have

$$
\left|K_{N}\right|^{1 / n} \simeq \frac{\sqrt{\log (N / n)}}{\sqrt{n}}, \quad\left|K_{N}^{\circ}\right|^{1 / n} \simeq \frac{1}{\sqrt{n \log (N / n)}}
$$

up to absolute constants.

\subsection{Mean Width}

Let $X$ be an $n$-dimensional normed space. Figiel and Tomczak-Jaegermann [14] defined the $\ell$-norm of $T \in L\left(\ell_{2}^{n}, X\right)$ by

$$
\ell(T)=\sqrt{n}\left(\int_{S^{n-1}}\|T y\|^{2} \sigma(d y)\right)^{1 / 2} .
$$

Equivalently, if $\left\{e_{j}\right\}$ is any orthonormal basis in $\mathbb{R}^{n}$, and if $g_{1}, \ldots, g_{n}$ are independent Gaussian random variables with distribution $N(0,1)$ on some probability space $\Omega$, we have

$$
\ell(T)=\left(\int_{\Omega}\left\|\sum_{i=1}^{n} g_{i}(\omega) T\left(e_{i}\right)\right\|^{2} d \omega\right)^{1 / 2}
$$


From well-known results of Lewis [24], Figiel and Tomczak-Jaegermann [14] and Pisier [29] it follows that for every $X=\left(\mathbb{R}^{n},\|\cdot\|\right)$ we can define a Euclidean structure $\langle\cdot, \cdot\rangle$ (called the $\ell$-structure) on $\mathbb{R}^{n}$, for which

$$
\ell\left(I: \ell_{2}^{n} \rightarrow X\right) \ell\left(I: \ell_{2}^{n} \rightarrow X^{*}\right) \leq c n \log \left[d\left(X, \ell_{2}^{n}\right)+1\right],
$$

where $c>0$ is an absolute constant and $I$ denotes the identity operator. It is not hard to check that

$$
\ell\left(I: \ell_{2}^{n} \rightarrow Z\right)=\int_{\Omega}\left\|\sum_{j=1}^{n} g_{j}(\omega) e_{j}\right\|_{Z} d \omega \simeq \sqrt{n} w\left(B_{Z}^{\circ}\right),
$$

for every $n$-dimensional space $Z$, and, hence, (2.14) is equivalent to the following fact: for every symmetric convex body $K$ in $\mathbb{R}^{n}$ there exists a linear image $\tilde{K}=T(K)$, $T \in G L(n)$, of $K(\tilde{K}$ is often called the " $\ell$-position" of $K$ ) for which

$$
w(\tilde{K}) w\left(\tilde{K}^{\circ}\right) \leq c \log \left[d\left(X_{K}, \ell_{2}^{n}\right)+1\right] .
$$

In view of Urysohn's inequality which states that for every convex body $K$ in $\mathbb{R}^{n}$,

$$
w(K) \geq 2\left(\frac{|K|}{\left|B_{2}^{n}\right|}\right)^{1 / n} \geq c \sqrt{n}|K|^{1 / n},
$$

where $c>0$ is an absolute constant, (2.16) and John's theorem show that, up to a $\log n$ term, a body which is in $\ell$-position has the "minimal possible mean width": $\tilde{K}$ satisfies the inequality

$$
w(\tilde{K}) \leq c^{\prime} \sqrt{n} \log n|\tilde{K}|^{1 / n} .
$$

In this subsection we get similar upper bounds for the mean width of a random $K_{N}$ and $K_{N}^{\circ}$.

Assume that $N \geq n(\log n)^{2}$. Starting with $K_{N}$, we write $x_{j}=\sqrt{n} u_{j}$ where $u_{j} \in S^{n-1}$, $j \leq N$, and hence

$$
w\left(K_{N}\right)=\int_{S^{n-1}} \max _{j \leq N}\left|\left\langle x_{j}, \theta\right\rangle\right| \sigma(d \theta)=\sqrt{n} \int_{S^{n-1}} \max _{j \leq N}\left|\left\langle u_{j}, \theta\right\rangle\right| \sigma(d \theta) .
$$

Now, by the spherical isoperimetric inequality we have

$$
\sigma\left(\theta:\left|\left\langle u_{j}, \theta\right\rangle\right| \geq c t / \sqrt{n}\right) \leq \exp \left(-t^{2}\right)
$$

for large $t$ (see [26]), which implies

$$
\int_{S^{n-1}} \max _{j \leq N}\left|\left\langle u_{j}, \theta\right\rangle\right| \sigma(d \theta) \leq c_{1} \frac{\sqrt{\log N}}{\sqrt{n}}
$$

therefore $w\left(K_{N}\right) \leq c_{2} \sqrt{\log N}$. Note that by Urysohn's inequality and the volume estimate in Proposition 2.3,

$$
c_{3} \sqrt{\log (N / n)} \leq w\left(K_{N}\right) \leq c_{2} \sqrt{\log N}
$$


for a random $K_{N}$. For the mean width of $K_{N}^{\circ}$ we use Theorem 2.2. Since $K_{N} \supset$ $(c \sqrt{\log (N / n)} / \sqrt{n}) Q_{n}$, we have

$$
h_{K_{N}^{\circ}}(\theta)=\|\theta\|_{K_{N}} \leq \frac{c_{4} \sqrt{n}}{\sqrt{\log (N / n)}}\|\theta\|_{\infty}
$$

therefore

$$
w\left(K_{N}^{\circ}\right) \leq \frac{c_{4} \sqrt{n}}{\sqrt{\log (N / n)}} \int_{S^{n-1}} \max _{i \leq n}\left|\theta_{i}\right| \sigma(d \theta) \simeq \frac{\sqrt{\log n}}{\sqrt{\log (N / n)}} .
$$

This is again close to the lower bound, apart from the $\sqrt{\log n}$ term. In particular,

$$
w\left(K_{N}\right) w\left(K_{N}^{\circ}\right) \leq c_{5} \frac{\sqrt{\log N}}{\sqrt{\log (N / n)}} \sqrt{\log n},
$$

that is $K_{N}$ satisfies an inequality analogous to (2.16). This fact will be later used in Banach-Mazur distance estimates.

Proposition 2.4. If $n \geq n_{0}$ and $N \geq n(\log n)^{2}$, then for a random $K_{N}$ we have

$$
c \sqrt{\log (N / n)} \leq w\left(K_{N}\right) \leq c^{\prime} \sqrt{\log N}
$$

and

$$
\frac{c}{\sqrt{\log (N / n)}} \leq w\left(K_{N}^{\circ}\right) \leq \frac{c^{\prime} \sqrt{\log n}}{\sqrt{\log (N / n)}},
$$

where $c, c^{\prime}>0$ are absolute constants.

\section{Asymptotic Properties of $\boldsymbol{X}_{N}$}

Theorem A provides enough information on the geometry of the unit ball of $X_{N}$. In fact, $K_{N}$ and $K_{N}^{\circ}$ belong to a rather restricted class of random convex bodies, and this allows us to determine several asymptotic parameters of the corresponding spaces.

\subsection{Unconditionality Properties of $X_{N}$}

We first show that unconditionality properties of $X_{N}$ are of the worst possible order as $N$ decreases to $n$. This fact is expected in view of well-known results from [13] and [1] about random proportional sections of $\ell_{\infty}^{m}$ which exhibit the same pathology. The source of our estimates is Lemma 2.1 which is the analogue of Kashin's theorem [23] in our context. However, our information on a random $K_{N}$ allows us to give an estimate for the full range of values of $N$.

Recall that an $n$-dimensional normed space $Y$ has 1-unconditional basis if there exists a basis $\left\{e_{1}, \ldots, e_{n}\right\}$ of $Y$ with the property

$$
\left\|\sum_{i=1}^{n} t_{i} e_{i}\right\|_{Y}=\left\|\sum_{i=1}^{n}\left|t_{i}\right| e_{i}\right\|_{Y}
$$

for every choice of reals $t_{1}, \ldots, t_{n}$. 
Theorem 3.1. If $N \geq c \log \left(\delta^{-1}\right) n$, then $X_{N} \in \mathcal{B}_{N}$ satisfies, with probability greater than $1-\delta$,

$$
d\left(X_{N}, Y\right) \geq \frac{c \sqrt{n}}{\sqrt{\log (2 N / n)}}
$$

for every $n$-dimensional normed space $Y$ with a 1-unconditional basis.

Proof. Consider the identity operator $I: X_{N}^{*} \rightarrow \ell_{2}^{n}$. Recall (see [28]) that the 1summing norm $\pi_{1}\left(T: Y^{*} \rightarrow \ell_{2}^{n}\right)$ of an operator $T: Y^{*} \rightarrow \ell_{2}^{n}$ is the minimum of all positive constants $A$ with the following property: for every $m \in \mathbb{N}$ and every choice of vectors $z_{1}, \ldots, z_{m} \in Y^{*}$,

$$
\sum_{j=1}^{m}\left\|T z_{j}\right\|_{2} \leq A \sup _{y \in B_{Y}} \sum_{j=1}^{m}\left|\left\langle y, z_{j}\right\rangle\right| .
$$

We first prove the following claim:

Claim 1. If $x_{1}, \ldots, x_{N}$ satisfy the conclusion of Lemma 2.1 , then $\pi_{1}(I) \simeq 1$.

Proof. Let $z_{1}, \ldots, z_{m} \in X_{N}^{*}$. Using Lemma 2.1, we write

$$
\sum_{j=1}^{m}\left\|z_{j}\right\|_{2} \leq \frac{1}{c_{1}} \cdot \frac{1}{N} \sum_{i=1}^{N} \sum_{j=1}^{m}\left|\left\langle x_{i}, z_{j}\right\rangle\right| \leq \frac{1}{c_{1}} \cdot \sup _{y \in K_{N}} \sum_{j=1}^{m}\left|\left\langle y, z_{j}\right\rangle\right|,
$$

therefore, $\pi_{1}(I) \leq c_{1}^{-1}$. On the other hand, by the definition of $\pi_{1}(I)$ we must also have

$$
\frac{1}{N} \sum_{j=1}^{N}\left\|x_{j}\right\|_{2} \leq \pi_{1}(I) \sup _{y \in K_{N}} \frac{1}{N} \sum_{j=1}^{N}\left|\left\langle y, x_{j}\right\rangle\right| .
$$

From the upper estimate in Lemma 2.1 we get

$$
\sup _{y \in K_{N}} \frac{1}{N} \sum_{j=1}^{N}\left|\left\langle y, x_{j}\right\rangle\right| \leq c_{2} \sup _{y \in K_{N}}\|y\|_{2} .
$$

Since $K_{N} \subseteq Q_{n} \subseteq \sqrt{n} B_{2}^{n}$ and $\left\|x_{j}\right\|_{2}=\sqrt{n}$ for every $j=1, \ldots, N$, we conclude that $\sqrt{n} \leq c_{2} \pi_{1}(I) \sqrt{n}$, which proves our claim.

Claim 2. Let $Q$ be a parallelepiped contained in $K_{N}^{\circ}$. Then

$$
|Q|^{1 / n} \leq \frac{2 \pi_{1}(I)}{n} .
$$

Proof. This fact was proved by Ball [1]. We include the argument for self-completeness. Consider a linear map $S: \mathbb{R}^{n} \rightarrow \mathbb{R}^{n}$ which takes $B_{\infty}^{n}$ onto $Q$. Applying Hadamard's 
inequality, the arithmetic-geometric means inequality and the definition of $\pi_{1}\left(S: \ell_{\infty}^{n} \rightarrow\right.$ $\left.\ell_{2}^{n}\right)$, we have

$$
\begin{aligned}
|Q|^{1 / n} & =2|\operatorname{det} S|^{1 / n} \leq 2\left(\prod_{i=1}^{n}\left\|S e_{i}\right\|_{2}\right)^{1 / n} \leq \frac{2}{n} \sum_{i=1}^{n}\left\|S e_{i}\right\|_{2} \\
& \leq \frac{2}{n} \pi_{1}\left(S: \ell_{\infty}^{n} \rightarrow \ell_{2}^{n}\right) \cdot \sup _{y \in B_{1}^{n}} \sum_{i=1}^{n}\left|\left\langle y, e_{i}\right\rangle\right| \\
& =\frac{2 \pi_{1}\left(S: \ell_{\infty}^{n} \rightarrow \ell_{2}^{n}\right)}{n} .
\end{aligned}
$$

Since

$$
\pi_{1}\left(S: \ell_{\infty}^{n} \rightarrow \ell_{2}^{n}\right) \leq\left\|S: \ell_{\infty}^{n} \rightarrow X_{N}^{*}\right\| \cdot \pi_{1}\left(I: X_{N}^{*} \rightarrow \ell_{2}^{n}\right),
$$

the result follows because $S\left(B_{\infty}^{n}\right)=Q \subseteq K_{N}^{\circ}$.

We can now complete the proof of Theorem 3.1. Let $Y$ be an $n$-dimensional space with 1-unconditional basis. If $d=d\left(X_{N}^{*}, Y\right)$, we may assume that $d B_{Y} \supseteq K_{N}^{\circ} \supseteq B_{Y}$. From a result of Losanovskii (see Chapter 3 of [30]), we can find a parallelepiped $Q \subseteq B_{Y}$ with $\left|B_{Y}\right| /|Q| \leq n^{n} / n$ !. Then, using our two claims, we get

$$
\left|K_{N}^{\circ}\right|^{1 / n} \leq d\left|B_{Y}\right|^{1 / n} \leq c d|Q|^{1 / n} \leq c^{\prime} d / n
$$

Now, Lemma 2.4 implies that $d\left(X_{N}^{*}, Y\right)=d \geq c^{\prime \prime} \sqrt{n} / \sqrt{\log (2 N / n)}$, and the theorem follows by duality.

Remark. A suitable choice of $\lambda>1$ shows the existence of a space $X_{N}$ with $N=\lambda n$ for which $d\left(X_{N}, Y\right) \geq c \sqrt{n}$ for every space $Y$ with 1-unconditional basis.

Let $N \geq n(\log n)^{2}$. Theorem 2.2 shows that $d\left(X_{N}, \ell_{\infty}^{n}\right) \leq c \sqrt{n} / \sqrt{\log (2 N / n)}$ for a random $X_{N}$. Combining this fact with Theorem 3.1, we see that $\ell_{\infty}^{n}$ is the space with 1-unconditional basis which is "closest" to $X_{N}$.

Theorem 3.2. If $N \geq n(\log n)^{2}$, then for a random $X_{N}$ we have

$$
d\left(X_{N}, \mathcal{U}\right) \simeq d\left(X_{N}, \ell_{\infty}^{n}\right) \simeq \frac{\sqrt{n}}{\sqrt{\log (N / n)}},
$$

where $\mathcal{U}$ is the class of $n$-dimensional spaces with 1-unconditional basis.

\subsection{Banach-Mazur Distance Estimates}

Propositions 2.1 and 2.4 indicate that the geometric distance between $K_{N}$ and $B_{2}^{n}$ and the mean width of $K_{N}$ are simultaneously controlled for a random $X_{N}$. This allows us to use the method of random orthogonal factorizations (which has its origin in work of 
Tomczak-Jaegermann [36], and was later developed in [5] and [11]) in order to estimate from above Banach-Mazur distances from a random $X_{N}$ to various classes of spaces.

The main point of the above method is the following result of Benyamini and Gordon [5], which makes use of an inequality of Chevet [10].

Lemma 3.1. Let $X$ and $Y$ be two $n$-dimensional normed spaces. Then

$$
\begin{aligned}
d(X, Y) \leq & \frac{c}{n}\left[\left\|I: X \rightarrow \ell_{2}^{n}\right\| \ell\left(I: \ell_{2}^{n} \rightarrow Y\right)+\left\|I: \ell_{2}^{n} \rightarrow Y\right\| \ell\left(I: \ell_{2}^{n} \rightarrow X^{*}\right)\right] \\
& \times\left[\left\|I: Y \rightarrow \ell_{2}^{n}\right\| \ell\left(I: \ell_{2}^{n} \rightarrow X\right)+\left\|I: \ell_{2}^{n} \rightarrow X\right\| \ell\left(I: \ell_{2}^{n} \rightarrow Y^{*}\right)\right],
\end{aligned}
$$

where $c>0$ is an absolute constant.

We apply this method to estimate the distance $d\left(X_{N}, X_{N}^{*}\right)$. The best known general estimate on this question is due to Bourgain and Milman [7] who have proved that

$$
d\left(X, X^{*}\right) \leq c n^{5 / 6} \log ^{\beta} n
$$

for every $n$-dimensional normed space $X$. The proof of (3.8) is again based on random orthogonal factorizations. If $X$ has a 1-unconditional basis or enough symmetries, then it gives a much better bound of the order of $\sqrt{n} \log ^{\beta} n$.

As we will see, despite the lack of unconditionality exhibited by $X_{N}$, we have a bound of this order for $d\left(X_{N}, X_{N}^{*}\right)$.

Theorem 3.3. There exists an absolute constant $C>0$ such that $d\left(X_{N}, X_{N}^{*}\right) \leq$ $C \sqrt{n \log n}$ for any $N \geq n$ and a random $X_{N}$.

Proof. We apply Lemma 3.1 with $X=X_{N}$ and $Y=X_{N}^{*}$. Taking into account (2.15), we get

$$
d\left(X_{N}, X_{N}^{*}\right) \leq c\left\|I: X_{N} \rightarrow \ell_{2}^{n}\right\| \cdot\left\|I: \ell_{2}^{n} \rightarrow X_{N}\right\| \cdot w\left(K_{N}\right) w\left(K_{N}^{\circ}\right) .
$$

We first consider the case $N \geq n^{2}$. By Proposition 2.4, for a random $K_{N}$ we have

$$
w\left(K_{N}\right) \leq c_{2} \sqrt{\log N}, \quad w\left(K_{N}^{\circ}\right) \leq \frac{c_{3} \sqrt{\log n}}{\sqrt{\log N}} .
$$

Since $K_{N} \subseteq Q_{n} \subseteq \sqrt{n} B_{2}^{n}$, we have

$$
\left\|I: X_{N} \rightarrow \ell_{2}^{n}\right\|=\max _{x \in K_{N}}\|x\|_{2} \leq \sqrt{n}
$$

and by Proposition 2.1 we have $c B_{2}^{n} \subseteq K_{N}$ for a random $K_{N}$, therefore

$$
\left\|I: \ell_{2}^{n} \rightarrow X_{N}\right\|=\max _{x \in B_{2}^{n}}\|x\|_{K_{N}} \leq c^{-1} .
$$

Combining the above, we get

$$
d\left(X_{N}, X_{N}^{*}\right) \leq C \sqrt{n \log n}
$$

for a random $X_{N}, N \geq n^{2}$. 
If $N \leq n^{2}$, we argue in a different way. Since $K_{N}$ has $n^{\alpha}$ vertices and $K_{N}^{\circ}$ has $n^{\beta}$ facets, with $\alpha=\beta \leq 2$, we can employ a well-known estimate from [16] to get

$$
d\left(X_{N}, X_{N}^{*}\right) \leq c \sqrt{n \log n} .
$$

Hence, we can find $C>0$ such that, independently of $N, d\left(X_{N}, X_{N}^{*}\right) \leq C \sqrt{n \log n}$ for a random $X_{N}$.

\subsection{Isotropic Constants}

Recall the definition of the isotropic position of a convex body $W$ in $\mathbb{R}^{n}$. There exists $T_{0} \in G L(n)$ such that the body $\tilde{W}=T_{0}(W)$ has volume 1 and satisfies the isotropic condition

$$
\int_{\tilde{W}}\langle x, \theta\rangle^{2} d x=L_{W}^{2}
$$

for every $\theta \in S^{n-1}$ (see [25] for a detailed account on this topic). This position is unique up to orthogonal transformations, therefore $L_{W}$ is an invariant of the linear class of $W$, and it is called the isotropic constant of $W$. One can check that the isotropic position of $W$ minimizes the quantity

$$
\frac{1}{|T(W)|^{1+2 / n}} \int_{T(W)}\|x\|_{2}^{2} d x
$$

over all $T \in G L(n)$. In particular,

$$
n L_{W}^{2} \leq \frac{1}{|W|^{1+2 / n}} \int_{W}\|x\|_{2}^{2} d x
$$

It is conjectured that there exists an absolute constant $C>0$ such that $L_{W} \leq C$ for every $n \in \mathbb{N}$ and every convex body $W$ in $\mathbb{R}^{n}$. The best known general estimate is due to Bourgain [6] who proved that $L_{W} \leq c \sqrt[4]{n} \log n$ for every symmetric convex body $W$. The conjecture is related to the slicing problem, which asks if there exists an absolute constant $c>0$ such that every convex body with volume 1 has a hyperplane section whose volume exceeds $c$. The connection comes from the fact that

$$
c_{1} \leq L_{W} \cdot\left|W \cap \theta^{\perp}\right| \leq c_{2}
$$

for every $\theta \in S^{n-1}$ and every isotropic convex body $W$, where $c_{1}, c_{2}>0$ are absolute constants (see [25]).

In this subsection we give upper bounds for the isotropic constant of $K_{N}$ and $K_{N}^{\circ}$. We make use of the following lemma [25].

Lemma 3.2. Let $W$ be a symmetric convex body in $\mathbb{R}^{n}$. Then

$$
L_{W} \leq \frac{c}{n} \cdot \frac{1}{|W|^{1+1 / n}} \int_{W}\|x\|_{1} d x .
$$

Also, $L_{W} \leq c d\left(X_{W}, Y\right)$ for every $Y \in \mathcal{U}$. 
Theorem 3.4. Let $N \geq n(\log n)^{2}$. With probability greater than $1-e^{-n}$ we have $L_{K_{N}^{\circ}} \leq C$, where $C>0$ is an absolute constant.

Proof. By Theorem 2.2, with probability greater than $1-e^{-n}$ we have $K_{N} \supseteq$ $(c \sqrt{\log (N / n)} / \sqrt{n}) Q_{n}$, where $c>0$ is an absolute constant. It follows that

$$
\|x\|_{1} \leq \frac{c_{1} \sqrt{n}}{\sqrt{\log (N / n)}}\|x\|_{K_{N}^{\circ}}
$$

for all $x \in \mathbb{R}^{n}$, where $c_{1}=1 / c$. Using Lemma 3.2 we get

$$
L_{K_{N}^{\circ}} \leq \frac{c_{2}}{\sqrt{n \log (N / n)}} \frac{1}{\left|K_{N}^{\circ}\right|^{1+1 / n}} \int_{K_{N}^{\circ}}\|x\|_{K_{N}^{\circ}} d x \leq \frac{c_{2}}{\sqrt{n \log (N / n)}\left|K_{N}^{\circ}\right|^{1 / n}} .
$$

In view of Lemma 2.4, the proof is complete.

Remark. Junge [22] has proved that if $X$ is an $n$-dimensional subspace of an $N$ dimensional space with 1-unconditional basis, then

$$
L_{B_{X}} \leq c \sqrt{\log (2 N / n)}
$$

for some absolute constant $c>0$. This estimate applies to any symmetric convex body with $N$ facets. Using this result for $\mathcal{B}_{N}^{*}$ when $n \leq N \leq n(\log n)^{2}$, we may summarize as follows:

Corollary 3.1. There exist absolute constants $c, C>0$ with the following property:

(a) If $n \leq N \leq n(\log n)^{2}$, then $L_{K_{N}^{\circ}} \leq c \sqrt{\log (2 N / n)} \leq C \sqrt{\log \log n}$.

(b) If $N \geq n(\log n)^{2}$, then $L_{K_{N}^{\circ}} \leq C$ for a random $K_{N}^{\circ}$.

Observe that we have a $\sqrt{\log \log n}$ estimate for a random $X_{N}^{*} \in \mathcal{B}_{N}^{*}$, which holds true in the full range of values of $N$.

We conclude this article with some simple estimates for the isotropic constant of $K_{N}$.

Proposition 3.1. Let $N \geq n(\log n)^{2}$. For a random $K_{N}$ we have

$$
L_{K_{N}} \leq C \frac{\min \{\log N, \sqrt{n}\}}{\sqrt{\log (N / n)}}
$$

where $C>0$ is an absolute constant.

Proof. Since $d\left(X_{N}, \ell_{\infty}^{n}\right) \simeq \sqrt{n} / \sqrt{\log (N / n)}$ for a random $X_{N}$, the estimate $L_{K_{N}} \leq$ $c_{1} \sqrt{n} / \sqrt{\log (N / n)}$ is an immediate consequence of Lemma 3.2.

If $N$ is not too big, then one can argue in a different way: consider the external volume ratio $\operatorname{evr}(W)=\inf (|E| /|W|)^{1 / n}$ of $W$, where the infimum is taken over all ellipsoids $E$ which contain $W$. Then we have the following. 
Claim. Let $z_{1}, \ldots, z_{N} \in \mathbb{R}^{n}$. If $W=\operatorname{co}\left\{ \pm x_{1}, \ldots, \pm x_{N}\right\}$, then

$$
L_{W} \leq c \log N \frac{\operatorname{evr}(W)}{\sqrt{n}},
$$

where $c>0$ is an absolute constant.

Proof. The formulation of the claim is invariant under linear transformations, therefore we may assume that $W$ is isotropic. Let $E$ be the ellipsoid of minimal volume which contains $W$. There exists a symmetric and positive $T \in G L(n)$ such that $T(E)=B_{2}^{n}$. Then

$$
\int_{W}\langle T x, x\rangle d x=[\operatorname{tr}(T)] L_{W}^{2} \geq n L_{W}^{2}|\operatorname{det} T|^{1 / n} .
$$

The equality comes from the isotropic condition (3.15) and the inequality is the arithmetic-geometric means inequality for the eigenvalues of $T$. On the other hand,

$$
\begin{aligned}
\int_{W}\langle T x, x\rangle d x & \leq \int_{W}\|T x\|_{W^{\circ}} d x=\int_{W} \max _{j \leq N}\left|\left\langle z_{j}, T x\right\rangle\right| d x \\
& =\int_{W} \max _{j \leq N}\left|\left\langle T z_{j}, x\right\rangle\right| d x .
\end{aligned}
$$

Since the $\psi_{1}$-norm of linear functionals on $W$ is equivalent to their 1-norm (see [25]), we get

$$
\int_{W} \max _{j \leq N}\left|\left\langle T z_{j}, x\right\rangle\right| d x \leq(c \log N) L_{W} \cdot \max _{j \leq N}\left\|T z_{j}\right\|_{2} .
$$

Since $z_{j} \in E$, we have $\left\|T z_{j}\right\|_{2} \leq 1, j=1, \ldots, N$. It follows that

$$
n|\operatorname{det} T|^{1 / n} L_{W} \leq c \log N
$$

and the result follows from $|\operatorname{det} T|^{-1 / n}\left|B_{2}^{n}\right|^{1 / n}=|E|^{1 / n}=\operatorname{evr}(W)$.

We can now show that $L_{K_{N}} \leq c_{2} \sqrt{\log (N / n)}$ : we observe that $K_{N} \subseteq \sqrt{n} B_{2}^{n}$ and using the fact that $\left(c_{3} \sqrt{\log (N / n)} / \sqrt{n}\right) Q_{n} \subseteq K_{N}$ we get

$$
\operatorname{evr}\left(K_{N}\right) \leq \sqrt{n} \frac{\left|B_{2}^{n}\right|^{1 / n}}{\left|K_{N}\right|^{1 / n}} \leq \frac{c \sqrt{n}}{\sqrt{\log (N / n)}} .
$$

Then our claim completes the proof.

Remark. On the other hand, Junge [22] has proved that the unit balls of projections of $N$-dimensional spaces with 1-unconditional basis have isotropic constant bounded by $c \log N$. Since $K_{N}$ is the unit ball of a projection of $\ell_{1}^{N}$, we see that $L_{K_{N}} \leq c \log n$ if $N \leq n(\log n)^{2}$. 


\section{Acknowledgments}

We are indebted to the referees of the paper for references and ideas which helped us to simplify the proof of Theorem 2.2 and to clarify the range of the parameters for which it holds true.

\section{References}

1. K. M. Ball, Normed Spaces with a Weak Gordon-Lewis Property, Lecture Notes in Mathematics 1470 , Springer-Verlag, Berlin (1991), pp. 36-47.

2. K. Ball and A. Pajor, Convex bodies with few faces, Proc. Amer. Math. Soc. 110 (1990), 225-231.

3. I. Bárány and Z. Füredi, Computing the volume is difficult, Discrete Comput. Geom. 2 (1987), 319-326.

4. I. Bárány and A. Pór, On 0-1 polytopes with many facets, Adv. in Math. 161 (2001), 209-228.

5. Y. Benyamini and Y. Gordon, Random factorization of operators between Banach spaces, J. Analyse Math. 39 (1981), 45-74.

6. J. Bourgain, On the Distribution of Polynomials on High Dimensional Convex Sets, Lecture Notes in Mathematics 1469, Springer-Verlag, Berlin (1991), pp. 127-137.

7. J. Bourgain and V. D. Milman, Distances between normed spaces, their subspaces and quotient spaces, Integral Equations Operator Theory 9 (1986), 31-46.

8. J. Bourgain, J. Lindenstrauss and V. D. Milman, Approximation of zonoids by zonotopes, Acta Math. 162 (1989), 73-141.

9. B. Carl and A. Pajor, Gelfand numbers of operators with values in Hilbert spaces, Invent. Math. 94 (1988), 459-504.

10. S. Chevet, Séries de variables aléatoires à valeur dans $E \check{\otimes} F$. Application aux produits d'espaces de Wiener abstraits, Séminaire Maurey-Schwartz, Exp. 19, Ecole Polytechnique, Palaiseau (1977).

11. W. J. Davis, V. D. Milman and N. Tomczak-Jaegermann, The distance between certain $n$-dimensional spaces, Israel J. Math. 39 (1981), 1-15.

12. M. E. Dyer, Z. Füredi and C. McDiarmid, Volumes spanned by random points in the hypercube, Random Structures Algorithms 3 (1992), 91-106.

13. T. Figiel and W. B. Johnson, Large subspaces of $\ell_{\infty}^{n}$ and estimates of the Gordon-Lewis constants, Israel J. Math. 37 (1980), 92-112.

14. T. Figiel and N. Tomczak-Jaegermann, Projections onto Hilbertian subspaces of Banach spaces, Israel J. Math. 33 (1979), 155-171.

15. T. Figiel, S. Kwapien and A. Pelczynski, Sharp estimates for the constants of local unconditional structure of Minkowski spaces, Bull. Acad. Polon. Sci. 25 (1977), 1221-1226.

16. T. Figiel, J. Lindenstrauss and V. D. Milman, The dimension of almost spherical sections of convex bodies, Acta Math. 139 (1977), 53-94.

17. A. A. Giannopoulos and V. D. Milman, Concentration property on probability spaces, Adv. in Math. 156 (2000), 77-106.

18. E. D. Gluskin, The diameter of the Minkowski compactum is approximately equal to $n$, Functional Anal. Appl. 15 (1981), 72-73.

19. E. D. Gluskin, Finite dimensional analogues of spaces without basis, Dokl. Akad. Nauk USSR 216 (1981), 1046-1050.

20. E. D. Gluskin, Extremal properties of orthogonal parallelepipeds and their applications to the geometry of Banach spaces, Math. USSR-Sbo. 64 (1989), 85-96.

21. F. John, Extremum Problems with Inequalities as Subsidiary Conditions, Courant Anniversary Volume, Interscience, New York (1948), pp. 187-204.

22. M. Junge, Proportional subspaces of spaces with unconditional basis have good volume properties, in Geometric Aspects of Functional Analysis, Operator Theory: Advances and Applications (eds. J. Lindenstrauss and V. D. Milman), Vol. 77, Birkhäuser, Basel (1995), pp. 121-129.

23. B. S. Kashin, Sections of some finite-dimensional sets and classes of smooth functions, Izv. Akad. Nauk. SSSR Ser. Mat. 41 (1977), 334-351. 
24. D. R. Lewis, Ellipsoids defined by Banach ideal norms, Mathematika 26 (1979), 18-29.

25. V. D. Milman and A. Pajor, Isotropic Position and Inertia Ellipsoids and Zonoids of the Unit Ball of a Normed n-Dimensional Space, Lecture Notes in Mathematics 1376, Springer-Verlag, Berlin (1989), pp. 64-104.

26. V. D. Milman and G. Schechtman, Asymptotic Theory of Finite Dimensional Normed Spaces, Lecture Notes in Mathematics 1200, Springer-Verlag, Berlin (1986).

27. S. J. Montgomery-Smith, The distribution of Rademacher sums, Proc. Amer. Math. Soc. 109 (1990), 517-522.

28. A. Pietsch, Absolute $p$-summierende Abbildugen in normierten Raümen, Studia Math. 28 (1967), 333 353.

29. G. Pisier, Holomorphic semi-groups and the geometry of Banach spaces, Ann. of Math. 115 (1982), 375-392.

30. G. Pisier, The Volume of Convex Bodies and Banach Space Geometry, Cambridge Tracts in Mathematics 94, Cambridge University Press, Cambridge (1989).

31. G. Schechtman, Random embeddings of Euclidean spaces in sequence spaces, Israel J. Math. 40 (1981), 187-192.

32. S. J. Szarek, On Kashin's almost Euclidean orthogonal decomposition of $\ell_{1}^{n}$, Bull. Acad. Polon. Sci. 26 (1978), 691-694.

33. S. J. Szarek, The finite dimensional basis problem, with an appendix on nets of Grassman manifold, Acta Math. 159 (1983), 153-179.

34. S. J. Szarek, Spaces with large distance to $\ell_{\infty}^{n}$ and random matrices, Amer. J. Math. 112 (1990), 899-942.

35. S. J. Szarek and N. Tomczak-Jaegermann, On nearly Euclidean decompositions of some classes of Banach spaces, Compositio Math. 40 (1980), 367-385.

36. N. Tomczak-Jaegermann, The Banach-Mazur distance between the trace classes $C_{p}^{n}$, Proc. Amer. Math. Soc. 72 (1978), 305-308.

37. N. Tomczak-Jaegermann, Banach-Mazur Distances and Finite Dimensional Operator Ideals, Pitman Monographs 38, Pitman, London (1989).

Received September 2, 2000, and in revised form October 2, 2001, and November 7, 2001.

Online publication July 24, 2002. 